\title{
Quantification of adipose tissue insulin sensitivity
}

\author{
Esben Søndergaard, ${ }^{1,2,3}$ Michael D Jensen ${ }^{1}$
}

${ }^{1}$ Endocrine Research Unit, Mayo Clinic, Rochester, Minnesota, USA

${ }^{2}$ Department of Endocrinology and Internal Medicine, Aarhus University Hospital, Aarhus, Denmark

${ }^{3}$ The Danish Diabetes Academy

\section{Correspondence to}

Professor Michael D Jensen, Endocrine Research Unit, Mayo Clinic, 200 1st St SW, Rm 5-194 Joseph,

Rochester, MN 55905, USA; jensen@mayo.edu

Accepted 24 March 2016 Published Online First 12 April 2016

Copyright (C) 2016 American Federation for Medical Research

\section{ABSTRACT}

In metabolically healthy humans, adipose tissue is exquisitely sensitive to insulin. Similar to muscle and liver, adipose tissue lipolysis is insulin resistant in adults with central obesity and type 2 diabetes. Perhaps uniquely, however, insulin resistance in adipose tissue may directly contribute to development of insulin resistance in muscle and liver because of the increased delivery of free fatty acids to those tissues. It has been hypothesized that insulin adipose tissue resistance may precede other metabolic defects in obesity and type 2 diabetes. Therefore, precise and reproducible quantification of adipose tissue insulin sensitivity, in vivo, in humans, is an important measure. Unfortunately, no consensus exists on how to determine adipose tissue insulin sensitivity. We review the methods available to quantitate adipose tissue insulin sensitivity and will discuss their strengths and weaknesses.

In metabolically healthy humans, adipose tissue is exquisitely sensitive to insulin. Lipolysis is almost completely suppressed at insulin concentration as low as $50-100 \mathrm{pmol} / \mathrm{L}$. ${ }^{1}$ Similar to muscle and liver, adipose tissue lipolysis is insulin resistant in adults with central obesity and type 2 diabetes $^{1}$ (figure 1 ). It has been hypothesized that insulin adipose tissue resistance may precede other metabolic defects in obesity and type 2 diabetes because excess adipose tissue free fatty acid (FFA) release may directly contribute to development of insulin resistance in muscle and liver. ${ }^{2}$ Therefore, precise and reproducible quantification of adipose tissue insulin sensitivity, in vivo, in humans, is an important measure. Unfortunately, no consensus exists on how to determine adipose tissue insulin sensitivity. We review a range of methods available to quantitate adipose tissue insulin sensitivity and will discuss their strengths and weaknesses.

\section{MEASUREMENTS OF LIPOLYSIS}

Methods that rely on plasma concentrations of FFA (or glycerol) to derive adipose tissue insulin sensitivity are inherently less robust because of the between and within-person differences in FFA clearance as a function of $\operatorname{sex}^{3}$ and concentration. ${ }^{4}$ Therefore, isotopic tracer approaches are preferred to quantify lipolysis. Being the most prevalent FFAs in the circulation, palmitic and oleic acids are useful as tracers and provide comparable estimates of total FFA turnover. ${ }^{5}$
In addition, glycerol tracers have been used to measure lipolysis. In theory, glycerol tracers better reflect total lipolysis because it has been argued that fatty acids may be re-esterified within adipocytes; this remains to be shown in vivo. However, glycerol is also released from the hydrolysis of circulating triglycerides. ${ }^{6}$ Under insulin suppressed conditions, this can be a significant confounder. Furthermore, we argue that the FFA released from adipose tissue into circulation is the physiological relevant measure as FFA, not glycerol, contributes to the metabolic effects of adipose tissue. The microdialysis technique can measure regional adipose tissue lipolysis, but the nuances of the using approach lies outside the scope of this brief review.

\section{MULTISTEP INSULIN CLAMP}

The multistep insulin clamp in combination with a FFA tracer has been used for decades to determine the dose-response relationship between plasma insulin concentration and lipolysis rates. ${ }^{17}$ It can be performed using the pancreatic clamp approach using somatostatin to obtain insulin concentrations ranging from near zero to those achieving complete suppression of lipolysis. ${ }^{8}$ However, the low-dose insulin infusion rate has to be carefully selected to obtain suppression in the mid-portion of the suppression range. The very steep relationship between FFA release and plasma insulin concentrations is such that it is easier to linearize the function by logarithmic transformation of both $\mathrm{x}$ (insulin) and y (FFA flux) axes. ${ }^{9}$ This allows for easier calculation of the 'true' $\mathrm{IC}_{50}$ (insulin concentration required for a $50 \%$ suppression of lipolysis). A multistep clamp performed without somatostatin can also be used to calculate an $\mathrm{IC}_{50}$ from basal insulin concentrations, which might be referred to as a 'basal' FFA-IC 50 . The FFA-IC 50 is well correlated with other indices of metabolic health, including whole body insulin sensitivity, with regard to glucose disposal and triglyceride concentrations. ${ }^{9}$ The drawback is that the method is cumbersome and time consuming. A single study requires $6-8 \mathrm{~h}$ to obtain a steady state in FFA turnover at each insulin step, limiting the number of subjects that can be studied.

\section{SINGLE STEP HYPERINSULINAEMIC EUGLYCEMIC CLAMP}

A simpler approach for measuring adipose tissue insulin sensitivity is to use a single step, 


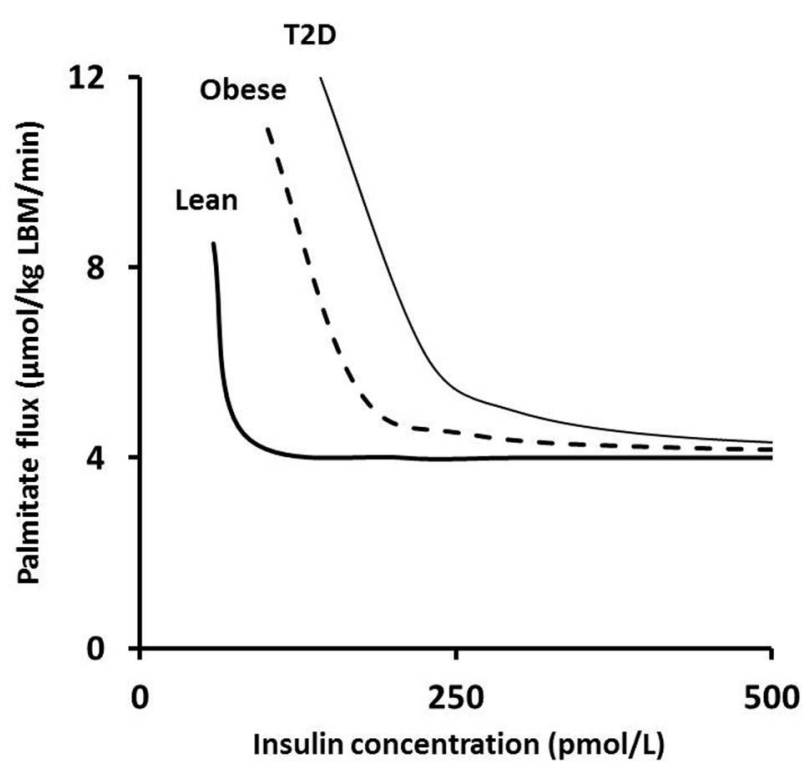

Figure 1 Relationship between insulin concentration and palmitate flux. Individual with obesity and type 2 diabetes are resistant to the effect of insulin on lipolysis. Lipolysis suppress to a similar level as for insulin sensitive individuals, but at a higher insulin concentration.

hyperinsulinemic, euglycemic clamp with isotopic tracer measures of lipolysis under basal and hyperinsulinemic conditions. This approach is considered the gold standard for quantifying whole body insulin sensitivity for glucose and is less time consuming than the multistep clamp. The single step insulin clamp can provide reasonable estimates of adipose tissue (and glucose) insulin sensitivity, again because the relationship between insulin concentration and FFA flux can be linearized using logarithmic transformation of insulin concentrations and flux. The two points (basal and insulin suppressed) obtained using this method can provide similar estimates of $\mathrm{ED}_{50}$ as the multistep clamp method. ${ }^{9}$ However, as can be observed in figure 1 , because the curve is very steep in insulin sensitive individuals, if the insulin dose selected is even slightly above that needed for maximal suppression, the $\mathrm{IC}_{50}$ can be grossly overestimated. $^{9}$

\section{MODELLING THE EFFECT OF INSULIN ON LIPOLYSIS}

Analog to the minimal model for quantification of the effect of insulin on glucose disposal, ${ }^{10}$ modelling approaches are available for the suppressive effect of insulin on FFA concentrations. ${ }^{11}$ Here, the effect of insulin on lipolysis is modelled during an insulin-modified frequently sampled intravenous glucose tolerance test (IM-FSIVGTT) ${ }^{11}$ or an oral glucose tolerance test ${ }^{12}$ without using an FFA tracer. For the IM-FSIVGTT, glucose concentration is maintained in at or above the euglycemic range, with a glucose infusion to avoid a hypoglycemia-induced counter-regulatory response, which itself can markedly alter lipolysis. ${ }^{13}$ These methods measure the dynamic response of FFA concentrations to a glucose bolus that stimulates endogenous insulin secretion. It has been argued that the dynamic insulin response to a glucose bolus is a more physiological challenge than the clamp method and that this approach may be able to detect differences in the adipose tissue response to insulin not revealed during constant insulin infusions. ${ }^{11}$ Our concern, however, is that these mathematical models rely on number assumptions regarding compartments and the kinetics of FFA clearance that have not been fully validated against models lacking such assumptions. Furthermore, high baseline FFA levels may negatively affect $\beta$-cell function and reduce the insulin response to the glucose bolus. ${ }^{14}$ Another limitation with model-based methods is their complexity. Researchers not trained in or lacking collaborators in mathematical modelling may have insufficient appreciation of the limitations and assumptions. This can lead to overinterpreting the results. In contrast, the clamp methods provide straightforward dose-response curves that are more easily interpreted.

\section{SIMPLE INDICES TO MEASURE ADIPOSE TISSUE INSULIN SENSITIVITY}

A simpler approach to measure adipose tissue insulin sensitivity is to measure postabsorptive plasma insulin and FFA concentrations (the Adipo-IR). This index is similar to the HOMA-IR, ${ }^{15}{ }^{16}$ and is calculated by multiplying postabsorptive concentrations of insulin and FFA. However, validation of the Adipo-IR against other measures of adipose tissue insulin sensitivity is lacking. Our concerns are that the relationship between insulin and FFA concentrations is affected by factors other than adipose tissue insulin sensitivity, such as age, sex, body composition, ethnicity or physical fitness. Furthermore, the index is based on a single measurement of insulin and FFA concentration, and is therefore more prone to analytical and day-to-day variation. The day-to-day variability of palmitate flux is substantial, as much as $31 \%$ in subjects consuming their usual diet. $^{8}$ This can be reduced to half by providing subjects with a weight maintaining diet, ${ }^{3}$ however, that will often not be applicable for larger studies where the Adipo-IR is applied. Whether the day-to-day variation in FFA concentration is independent of or follows the variation in insulin concentration remains unknown. The major advantage of this index is that it is easy to measure and calculate for large numbers of subjects. Therefore, it can be applied for studies with substantially larger sample size compared with that of the clamp method.

Another index of adipose tissue insulin sensitivity has been suggested, but has not yet been applied for any additional studies. The Adipose Tissue Insulin Resistance Index (ATIRI) multiplies postabsorptive palmitate flux rates determined by a palmitate tracer relative to postabsorptive insulin concentrations. ${ }^{17}$ This has been validated with a reasonable predictive value to suppression of lipolysis during a hyperinsulinemic euglycemic clamp $\left(\mathrm{R}^{2}=0.73\right)$ in obese individuals. ${ }^{17}$

\section{CONCLUSION}

Adipose tissue insulin sensitivity has attracted less attention than has insulin sensitivity in muscle and liver. However, the key role of adipose tissue in delivering FFA for other insulin sensitive tissues necessitates a better understanding of the regulation of lipolysis. Several methods are available to estimate adipose tissue insulin sensitivity. We argue that the most pharmacologically pure approach is the 'true' FFA-IC 50 that we described above, using somatostatin to 
obtain near zero insulin concentrations. How the other methods compare to this approach, and whether any of the methods is superior in term of predicting clinical outcomes, is unknown.

Contributors ES wrote the review and MDJ reviewed and edited the draft.

Funding This work was supported by National Institutes of Health grants, DK-45343, DK-40484 and DK-50456, and grants from The Danish Diabetes Academy supported by the Novo Nordisk Foundation, Danish Diabetes Association, Augustinus Foundation and the Research Council Aarhus University Hospital.

Competing interests None declared.

Provenance and peer review Commissioned; externally peer reviewed.

\section{REFERENCES}

1 Groop LC, Bonadonna RC, DelPrato S, et al. Glucose and free fatty acid metabolism in non-insulin-dependent diabetes mellitus. evidence for multiple sites of insulin resistance. J Clin Invest 1989;84:205-13.

2 Ferrannini E, Barrett EJ, Bevilacqua $\mathrm{S}$, et al. Effect of fatty acids on glucose production and utilization in man. J Clin Invest 1983;72:1737-47.

3 Nielsen S, Guo Z, Albu JB, et al. Energy expenditure, sex, and endogenous fuel availability in humans. J Clin Invest 2003;111:981-8.

4 Miles JM, Ellman MG, McClean KL, et al. Validation of a new method for determination of free fatty acid turnover. Am J Physiol 1987;252:E431-8.

5 Mittendorfer B, Liem O, Patterson BW, et al. What does the measurement of whole-body fatty acid rate of appearance in plasma by using a fatty acid tracer really mean? Diabetes 2003;52:1641-8.

6 Jensen MD, Chandramouli V, Schumann WC, et al. Sources of blood glycerol during fasting. Am J Physiol Endocrinol Metab 2001;281: E998-E1004.
7 Groop LC, Saloranta C, Shank M, et al. The role of free fatty acid metabolism in the pathogenesis of insulin resistance in obesity and noninsulin-dependent diabetes mellitus. J Clin Endocrinol Metab 1991;72:96-107.

8 Jensen MD, Caruso M, Heiling V, et al. Insulin regulation of lipolysis in nondiabetic and IDDM subjects. Diabetes 1989;38:1595-601.

9 Jensen MD, Nielsen $S$. Insulin dose response analysis of free fatty acid kinetics. Metab Clin Exp 2007;56:68-76.

10 Bergman RN, Ider $\mathrm{YZ}$, Bowden CR, et al. Quantitative estimation of insulin sensitivity. Am J Physiol 1979;236:E667-77.

11 Periwal V, Chow CC, Bergman RN, et al. Evaluation of quantitative models of the effect of insulin on lipolysis and glucose disposal. Am J Physiol Regul Integr Comp Physiol 2008;295:R1089-96.

12 Boston RC, Moate PJ. NEFA minimal model parameters estimated from the oral glucose tolerance test and the meal tolerance test. Am J Physiol Regul Integr Comp Physiol 2008;295:R395-403.

13 Thomaseth K, Brehm A, Pavan A, et al. Modeling glucose and free fatty acid kinetics during insulin-modified intravenous glucose tolerance test in healthy humans: role of counterregulatory response. Am I Physiol Regul Integr Comp Physiol 2014;307:R321-31.

14 Kashyap S, Belfort R, Gastaldelli A, et al. Asustained increase in plasma free fatty acids impairs insulin secretion in nondiabetic subjects genetically predisposed to develop type 2 diabetes. Diabetes 2003:52:2461-74.

15 Gastaldelli A, Harrison SA, Belfort-Aguilar R, et al. Importance of changes in adipose tissue insulin resistance to histological response during thiazolidinedione treatment of patients with nonalcoholic steatohepatitis. Hepatology 2009;50:1087-93.

16 Abdul-Ghani MA, Molina-Carrion M, Jani R, et al. Adipocytes in subjects with impaired fasting glucose and impaired glucose tolerance are resistant to the anti-lipolytic effect of insulin. Acta Diabetol 2008:45:147-50

17 Fabbrini E, Magkos F, Conte C, et al. Validation of a novel index to assess insulin resistance of adipose tissue lipolytic activity in obese subjects. I Lipid Res 2012;53:321-4. 